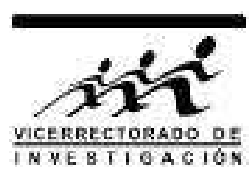

\title{
Evaluación de la concavidad de un espejo para telescopio mediante procesamiento de un solo interferograma
}

\author{
R. A. Montalvo*1 2, Mabel Tesillor, Ivan Choque ${ }^{2}$ y Miguel Asmad² \\ ${ }^{1}$ Facultad de Ciencias Físicas, Universidad Nacional Mayor de San Marcos, Lima, Perú \\ ${ }^{2}$ Sección Física, Departamento de Ciencias, Pontificia Universidad Católica del Perú, Lima, Perú
}

Recibido 25 enero 2015 - Aceptado 30 abril 2015

\begin{abstract}
En este trabajo se muestra que, a partir de un interferograma, es posible calcular el error de la forma cóncava para una superficie pulida ópticamente que será usada como espejo en un telescopio reflector. En el Laboratorio de Óptica de la PUCP, se ha implementado un interferómetro TwymanGreen donde se adquieren imágenes de la superficie, superpuestas con franjas de interferencia que se denominan interferogramas. Para realizar el cálculo se utilizan algoritmos de evaluación de la fase basados en la transformada de Fourier que permite reconstruir las imágenes procesadas para obtener la información de la forma de la superficie y su desviación respecto de su forma ideal cóncava.

Fueron evaluadas las imágenes de los interferogramas de una misma superficie para diferentes frecuencias de franjas; en cada caso, una sola imagen permite determinar el error superficial, sin tener contacto con la superficie.
\end{abstract}

Palabras claves: Perfilometría, transformada de Fourier, error de superficie cóncava.

\section{Testing concavity of a telescope mirror by processing of a single interferogram}

This paper shows that it is possible to estimate, from a single interferogram image, the error of the concave shape for an optically polished surface that will be used as a mirror in a reflecting telescope. In the Optics Laboratory of the PUCP, a Twyman-Green interferometer has been implemented; surface images with overlapping interference fringes known as interferograms are acquired. Algorithms based on Fourier transform allows the reconstruction of the surface shape and its deviation from ideal concave shape.

Images were evaluated for different numbers of interference fringes in the same surface; in each case, a single image was necessary to determine surface error with the added advantage of not having contact with the surface.

Keywords: Perfilometry, Fourier transform, error concave surface.

Los telescopios reflectores fueron diseñados desde finales del siglo XVII siendo su característica el uso de espejos en lugar de lentes; los espejos cóncavos en estos telescopios forman una imagen virtual más amplia que la real. En los sistemas ópticos las superficies de cada elemento óptico deben ser cuidadosamente elaboradas, la sola presencia de microrugosidades en sus superficies alteran la calidad de las imágenes finales [1,2]. Por este motivo en óptica de precisión, el control de la calidad superficial en todas la etapas de elaboración, permite obtener elementos ópticos de alta calidad con errores en su superficie, menores a décimas de fracción de la

${ }^{*}$ rmontalvob@unmsm.com.pe 
longitud de onda visible, respecto de su forma ideal.

Existen diferentes métodos para evaluar las superficies ópticas, muchos de estos son de contacto y pueden destruir la muestra. Dentro de los no destructivos, los métodos ópticos o métodos sin contacto como los interferométricos son los más empleados. Estos están basados en el análisis de las franjas de interferencia de dos haces. En este método, la posibilidad de cambiar la magnificación permite también obtener un amplio rango de resolución lateral y campo de medida, así como, mayor sensibilidad y precisión. En las técnicas interferométricas, el patrón de franjas o interferograma es el resultado del comportamiento físico de la muestra.

Los avances en las técnicas ópticas fue posible debido a las mejoras en los sistemas de adquisición de las imágenes que permitieron el proceso automático de las imágenes en un lapso de tiempo breve. En particular, el método de la transformada de Fourier [3] es aplicado a una imagen bidimensional permitiendo la extracción inmediata de la geometría de la superficies [4]. La transformada de Fourier es una herramienta importante dentro del procesamiento digital de las imágenes; esta es utilizada para descomponer una imagen en sus componentes seno y coseno. Cada punto de la imagen en el dominio de Fourier representa una frecuencia particular contenida en la imagen en el dominio del espacio. La transformada de Fourier de una imagen permite filtrar información solo de la fase, eliminando ruido. Obtenida la información en el dominio de las frecuencias, la operación inversa retorna los datos en el dominio espacial reconstruyendo la imagen. El método de la transformada de Fourier logra distinguir totalmente entre una depresión o elevación en la evaluación de la forma de la superficie del objeto ya que se obtiene la distribución de la altura de cada píxel para todo el relieve. También durante el experimento se puede verificar si se trata de una depresión o elevación por la dirección de desplazamiento de las franjas.

La imagen del interferograma es capturada mediante una cámara CMOS, cada punto se conoce como un elemento de imagen o píxel. La cámara digitaliza cada píxel a un valor entre 0 , negro y 255 , tono más brillante, através de toda una gama de grises que se encuentra en ese intervalo de valores. Las imágenes presentadas fueron evaluadas mediante algoritmos que permitieron extraer la información de la superficie.

\section{Experimento}

Se muestra en la Figura 1 el esquema del interferómetro de Twyman-Green, en el que un haz de luz laser de $\mathrm{He}-\mathrm{Ne}$, con una longitud $\lambda=632.8 \mathrm{~nm}$ y una potencia de $15 \mathrm{~mW}$ incide sobre un filtro $\mathrm{F}$ de densidad neutra que lo atenua y sigue sobre una lente $\mathrm{L} 1$, de distancia focal de $5 \mathrm{~mm}$, posteriormente el haz atraviesa una lente colimadora L2 de distancia focal de $180 \mathrm{~mm}$, luego atraviesa un divisor que genera dos haces, de iguales amplitudes. Uno de los haces, de referencia, se dirige al espejo plano referencial M1; el otro haz, objeto, se dirige a una lente convergente L3 y de allí al espejo M2 que en este caso es el espejo cóncavo que se pretende evaluar. Ambos haces se reflejan e interfieren; ésta interferencia es capturada por un CMOS de $1024 \times 1280$ píxels [5] para ser llevada al computador.

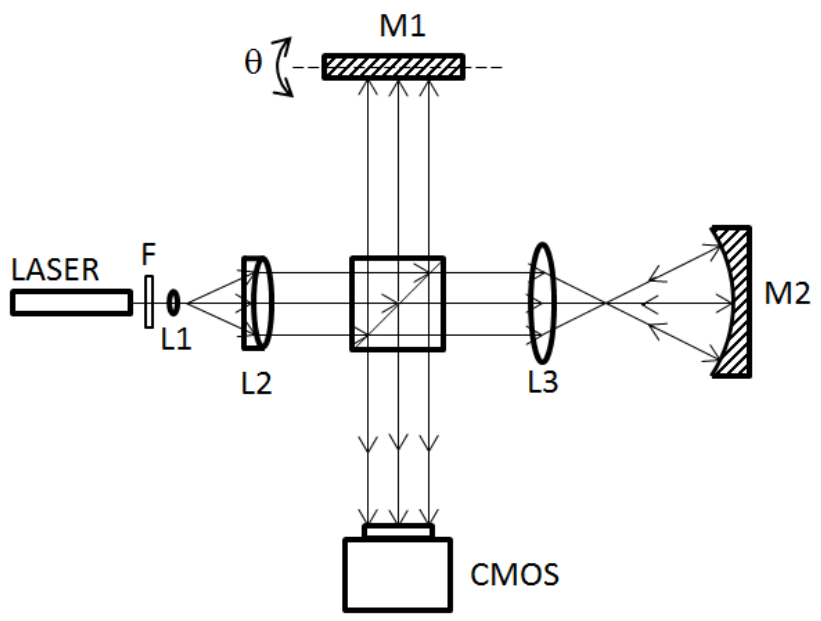

Figura 1: Arreglo experimental del Twyman Green para evaluar el espejo cóncavo M2.

El espejo cóncavo ha evaluar es descrito en el diagrama experimental como $\mathrm{M} 2$ que se muestra en la Figura 2 y posee un diámetro de $55 \mathrm{~mm}$. Realizando el experimento para este espejo cóncavo M2, se capturaron cuatro interferogramas para diferentes números de franjas de interferencia, que se consigue al variar el ángulo $\theta$ inclinando el espejo M1. En todas la imágenes, Figura 3, se aprecian franjas con distorsión central, producto de la variación del frente de onda plana de la superficie del espejo cóncavo. Si ambos frentes de onda provenientes de los espejos cóncavo y referencial fuesen perfectos, es decir, cóncavo y plano, respectivamente, el interferograma presentaría franjas rectas y paralelas; la desviación de la rectitud de las franjas brindan información de la desviación de la superficie respecto a su forma ideal cóncava. 


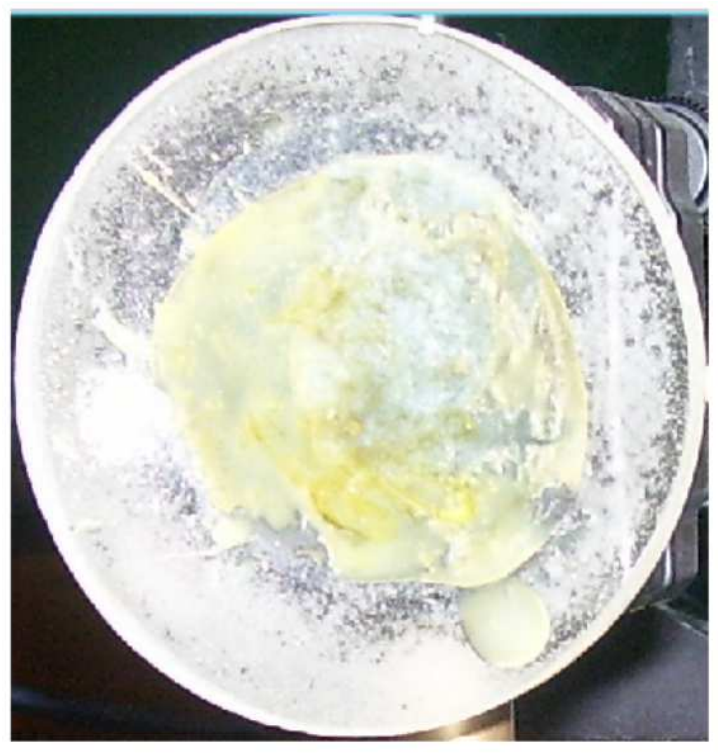

Figura 2: Espejo cóncavo a analizar identificado como M2 en el arreglo experimental.

Los interferogramas, Fig 3 muestran cualitativamente protuberancias o depresiones centrales, que el procesamiento de los interferogramas mediante la transformada de Fourier determina las mismas, cuantitativamente.

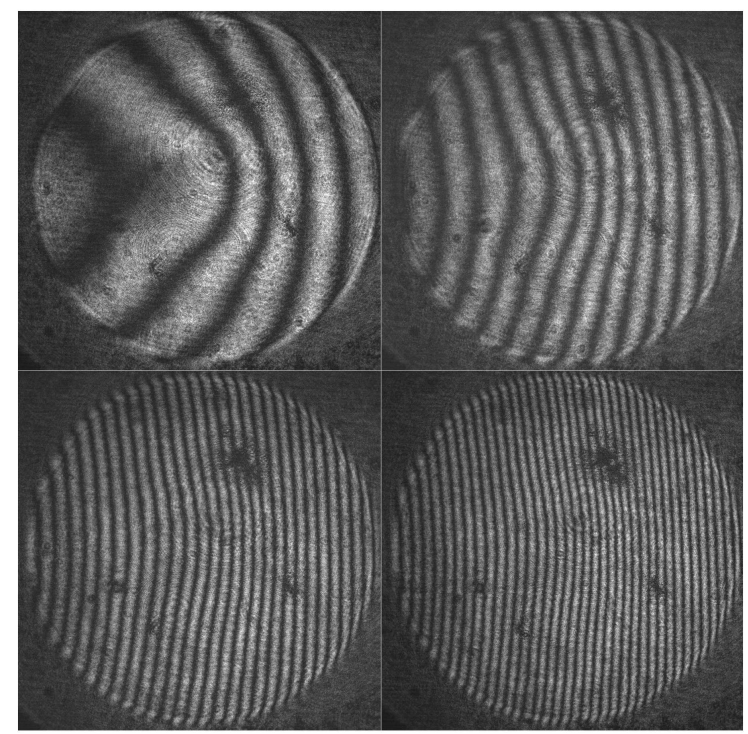

Figura 3: Se muestran los interferogramas correspondientes a $6,13,29$ y 39 franjas de interferencia, de izquierda a derecha y de arriba a abajo, respectivamente.

El número de franjas fue variado mediante la inclinación del espejo M1 que refleja el haz de referencia, un ángulo $\theta$. Los haces, referencial y objeto, al interferir son capturados por la cámara. La presencia de manchas oscuras en todas las imágenes de los interferogramas de la Figura 6, se deben a las condiciones internas de la cámara.

\section{Detalle teórico}

Aquí presentamos el detalle teórico involucrado en el diseño del experimento usando el interferómetro de Twyman-Green en la configuración explicada en la sección anterior.

La imagen adquirida presenta un patrón de franjas producto de la interferencia de los haces dirigidos al CMOS, la intensidad en un píxel de tamaño $p_{x} * p_{y}$ ubicado en $\left(x_{n}, y_{m}\right)=\left(n * p_{x}, m * p_{y}\right)$, donde $n \in[0,1023]$ y $m \in[0,1279]$, está dado por

$$
\begin{aligned}
& g\left(x_{n}, y_{n}\right)=a\left(x_{n}, y_{m}\right)+ \\
& \quad b\left(x_{n}, y_{m}\right) \cos \left[2 \pi f_{0}\left(x_{n}+y_{m}\right)+\phi\left(x_{n}, y_{n}\right)\right],
\end{aligned}
$$

donde $a\left(x_{n}, y_{m}\right)$ representa el background, $b\left(x_{n}, y_{m}\right)$ el contraste o visibilidad de las franjas brillantes y oscuras observadas, $f_{0}$ la frecuencia espacial de la portadora y $\phi\left(x_{n}, y_{m}\right)$ la fase que contiene la forma del objeto en estudio, en nuestro caso el espejo cóncavo M2. Los términos $a\left(x_{n}, y_{m}\right)$ y $b\left(x_{n}, y_{m}\right)$ son irradiancias que deben ser separadas de la fase para poder evaluarla; esto es posible debido a que $\phi\left(x_{n}, y_{m}\right), a\left(x_{m}, y_{m}\right), b\left(x_{n}, y_{m}\right)$ varían muy lentamente en comparación con la frecuencia de la portadora $f_{0}$.

Reescribiendo la Ec.(1) usando la identidad de Euler,

$$
\begin{aligned}
& g\left(x_{n}, y_{m}\right)=a\left(x_{n}, y_{m}\right)+ \\
& \frac{b\left(x_{n}, y_{m}\right)}{2}\left[e^{i\left[2 \pi f_{0}\left(x_{n}+y_{m}\right)+\phi\left(x_{n}, y_{m}\right)\right]}\right]+ \\
& \frac{b\left(x_{n}, y_{m}\right)}{2}\left[e^{-i\left[2 \pi f_{0}\left(x_{n}+y_{m}\right)+\phi\left(x_{n}, y_{m}\right)\right]}\right]
\end{aligned}
$$

y considerando que

$$
\begin{gathered}
c\left(x_{n}, y_{m}\right)=\frac{b\left(x_{n}, y_{m}\right)}{2}\left[e^{i \phi\left(x_{n}, y_{m}\right)}\right] \\
c^{*}\left(x_{n}, y_{m}\right)=\frac{b\left(x_{n}, y_{m}\right)}{2}\left[e^{-i \phi\left(x_{n}, y_{m}\right)}\right],
\end{gathered}
$$

observándose que $c^{*}\left(x_{n}, y_{m}\right)$ es el complejo conjugado de $c\left(x_{n}, y_{m}\right)$, y la Ec.(2) se expresa como

$$
\begin{array}{r}
g\left(x_{n}, y_{m}\right)=a\left(x_{n}, y_{m}\right)+c\left(x_{n}, y_{m}\right) e^{i 2 \pi f_{0}\left(x_{n}+y_{m}\right)}+ \\
c^{*}\left(x_{n}, y_{m}\right) e^{-i 2 \pi f_{0}\left(x_{n}+y_{m}\right)}
\end{array}
$$




\section{La transformada de Fourier}

Aplicando la transformada de Fourier a la Ec.(4) solo en la dirección espacial $x$, cada sumando de la Ec.(4) se expresa como

$$
\begin{aligned}
A\left(f_{l}, y_{m}\right) & =\frac{1}{N} \sum_{n=0}^{N-1} a\left(x_{n}, y_{m}\right) e^{i 2 \pi f_{l} x_{n}} \\
C\left(f_{l}+f_{0}, y_{m}\right) & =\frac{e^{i 2 \pi f_{0} y_{m}}}{N} \times \\
C^{*}\left(f_{l}-f_{0}, y_{m}\right) & =\frac{\sum_{n=0}^{N-1} c\left(x_{n}, y_{m}\right) e^{i 2 \pi\left(f_{l}+f_{0}\right) x_{n}}}{N} \times \\
& \sum_{n=0}^{N-1} c^{*}\left(x_{n}, y_{m}\right) e^{i 2 \pi\left(f_{l}-f_{0}\right) x_{n}},
\end{aligned}
$$

donde $l \in[0,1023]$ en el espacio de frecuencias. La función $g\left(x_{n}, y_{m}\right)$ en el dominio espacial es transformado al dominio de las frecuencias espaciales resumiendo las tres ecuaciones anteriores como

$$
\begin{aligned}
G\left(f_{l}, y_{m}\right)=A\left(f_{l}, y_{m}\right)+C & \left(f_{l}+f_{0}, y_{m}\right) \\
& +C^{*}\left(f_{l}-f_{0}, y_{m}\right)
\end{aligned}
$$

donde $C^{*}\left(f_{l}-f_{0}, y_{m}\right)$ es una notación que no necesariamente indica el complejo conjugado de $C\left(f_{l}+f_{0}, y_{m}\right)$.

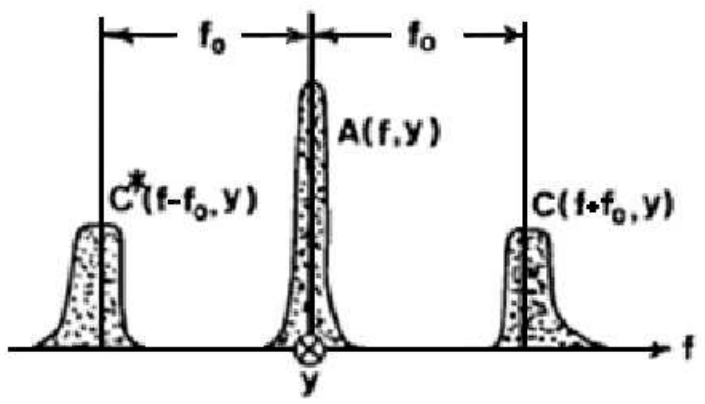

Figura 4: Aplicación de la transformada de Fourier; los parametros $A\left(f_{l}, y_{m}\right), C^{*}\left(f_{l}-f_{0}, y_{m}\right)$ y $C\left(f_{l}+f_{0}, y_{m}\right)$ están separados.

En la Figura 4 la función $G\left(f_{l}, y_{m}\right)$ se encuentra en el dominio de las frecuencias, $A\left(f_{l}, y_{m}\right)$ corresponde al background que ha sido separado y puede ser filtrado. Los dos lóbulos se encuentran a una distancia en frecuencia igual a dos veces la portadora $f_{0}$; ambos lóbulos no son conjugados en general, pero para cada valor de $l$ con $f_{0} \neq 0$ los son y contienen las informaciones de la superficie proporcionada por $C^{*}\left(f_{l}-f_{0}, y_{m}\right)$ у $C\left(f_{l}+f_{0}, y_{m}\right)$.

Se elige uno de los lóbulos y se traslada al centro del sistema de las frecuencias, Fig 5 , generalmente suele elegirse el derecho.

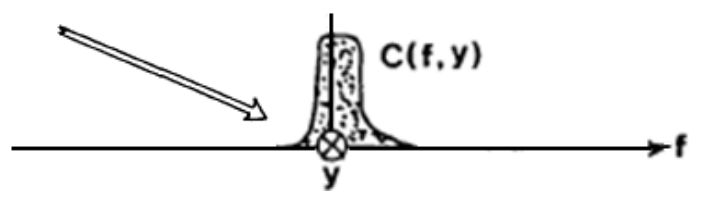

Figura 5: Desplazamiento del lóbulo que contiene la fase.

Una vez filtrada la función que contiene las informaciones de la superficie, se aplica la transformada inversa de Fourier a $C\left(f_{l}, y_{m}\right) \circ C^{*}\left(f_{l}, y_{m}\right)$ para obtener la función en el dominio espacial $c\left(x_{n}, y_{m}\right) \circ c^{*}\left(x_{n}, y_{m}\right)$, Ec.(3), donde $A, C, C^{*}$ son la transformada de Fourier de $a, c$ y $c^{*}$.

$$
c\left(x_{n}, y_{m}\right)=\frac{b\left(x_{n}, y_{m}\right)}{2}\left[e^{i \phi\left(x_{n}, y_{m}\right)}\right]
$$

\section{Fase envuelta}

De la Ec.(9), $\operatorname{Re}[c]=\frac{b}{2} \cos \phi$ e $\operatorname{Im}[c]=\frac{b}{2} \operatorname{sen} \phi$; la fase $\phi\left(x_{n}, y_{m}\right)$ se determina aplicando el arco tangente a la división de la parte imaginaria y real de $c$.

$$
\phi\left(x_{n}, y_{m}\right)=\arctan \left\{\frac{\operatorname{Im}\left[c\left(x_{n}, y_{m}\right)\right]}{\operatorname{Re}\left[c\left(x_{n}, y_{m}\right)\right]}\right\}
$$

El dominio del arco tangente se encuentra en el intervalo $\left(-\frac{\pi}{2}, \frac{\pi}{2}\right)$; mediante el signo del numerador y denominador se puede conocer el cuadrante al cual pertenece el ángulo por lo que la fase puede calcularse sin ambiguedad en el rango entre $-\pi \mathrm{y}+\pi$. Matlab tiene la función $\operatorname{atan} 2(y, x)$ que presenta discontinuidades o saltos de $2 \pi$, a este resultado se le conoce como la fase envuelta.

\section{Fase desenvuelta}

Desde hace dos décadas, desenvolver la fase ha sido un área de investigación bastante prolífico, y se ha observado que para determinadas situaciones resulta ser un problema complicado recuperar la continuidad de la fase, por ello, en la literatura se encuentran una gran variedad de algoritmos desarrollados para determinadas 
situaciones específicas. A partir de la Ec.(10), la fase presenta discontinuidades como se aprecia en la parte a) de la Figura 6, para resolver este problema se aplica un algoritmo que permite desenvolver la discontinuidad [6]. El algoritmo consiste en determinar el punto de discontinuidad, este proceso se logra sustrayendo cada valor de fase respecto a su vecino inmediato anterior, el salto se produce cuando la diferencia es menor a $-\pi$ o mayor a $+\pi$. La Ec.(11) representa el algoritmo basado en el método de Itoh [7].

$$
\Delta \phi_{n}=\phi_{n}-\phi_{n-1} \quad, \quad\left|\Delta \phi_{n}\right|<\pi
$$

Si la diferencia entre dos puntos es menor a $-\pi$, se produce un salto negativo $\downarrow$ luego se suma $+2 \pi$, Ec.(12), a este y a todos los puntos posteriores a esta discontinuidad hasta que se produzca una nueva discontinuidad. Si no se produce una nueva discontinuidad no se adiciona o sustrae nada. Si la diferencia es mayor a $+\pi$, salto positivo $\uparrow$, se resta $2 \pi$ al punto y tambien a todos los puntos siguientes de la discontinuidad, Ec.(13). El proceso continúa hasta que se produzca la siguiente discontinuidad en cuyo caso la suma o resta corresponde al siguiente entero $4 \pi$ y así se compensa la distribución de las discontinuidades, parte b) de la Figura [6.

$$
\begin{aligned}
& \text { Si } \phi_{n}-\phi_{n-1}<0 \text {, entonces } \phi_{n}=\phi_{n}+2 k \pi, \\
& \text { si } \phi_{n}-\phi_{n-1}>0 \text {, entonces } \phi_{n}=\phi_{n}-2 k \pi,
\end{aligned}
$$

donde $k$ indica el número de discontnuidades que se encuentran y la suma o sustraccioń de $2 k \pi$ produce la distribucion contínua de la fase como se observa en la parte c) de la Figura 6. Con este proceso se desenvuelve la fase y la información de la imagen filtrada es recuperada.

Debido a la diferencia de camino óptico de los haces para cada punto de la superficie, variaciones en la intensidad están presentes debido a las diferencias de fase [8], de esta manera el error de la superficie del espejo $\mathrm{M} 2$ respecto a su forma ideal cóncava es obtenida en cada punto a partir de la Ec.(14) [9].

$$
h\left(x_{n}, y_{m}\right)=\frac{\lambda}{4 \pi} \phi\left(x_{n}, y_{m}\right)
$$
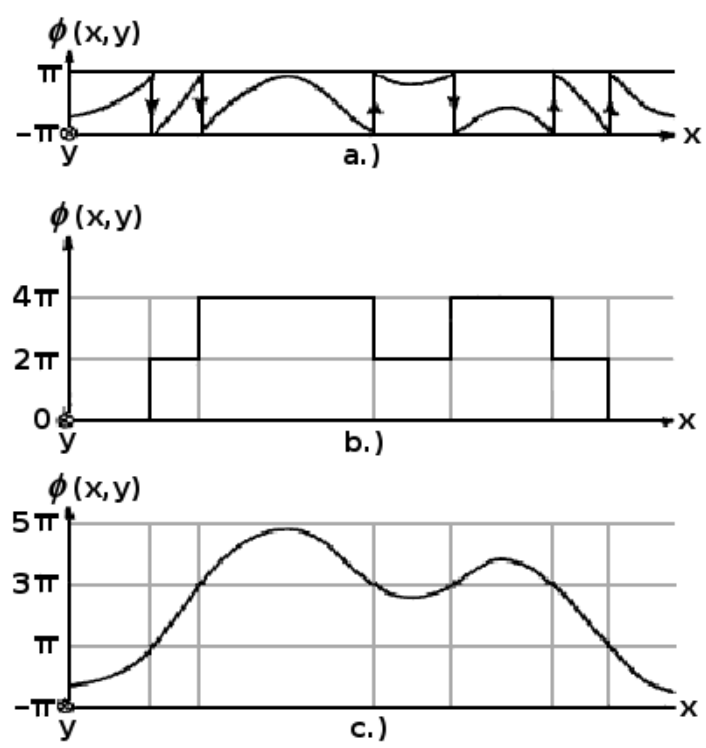

Figura 6: Desarrollo de la continuidad de la fase aplicando el algoritmo de desenvolvimiento de Itoh [5].

\section{Resultados}

En la Figura 7 se presenta uno de los interferogramas del espejo cóncavo que muestra el dominio espacial de las interferencias y a la derecha se muestra el dominio de las frecuencias obtenido mediante la transformada de Fourier. En la parte inferior se muestra un perfil de intensidad de la transformada de Fourier a lo largo de una línea que pasa por el máximo central.

En el dominio de las frecuencias, se elige uno de los lóbulos y se traslada a la ubicación del máximo, aislando la fase y la intensidad de la imagen. Aplicando la transformada inversa, Ec.(10), se recupera la información de la fase envuelta en el dominio espacial. En la Figura 8 , las cuatro imágenes $A_{1}, A_{2}, A_{3}$ y $A_{4}$ muestran la fase envuelta para los interferogramas de la superficie del espejo cóncavo M2 correspondientes a 6, 13, 29 y 39 franjas, respectivas a la Figura 6. La fase envuelta se aprecia por la variación abrupta claro-oscuro, que representa la discontinuidad en valores de fase $-\pi \mathrm{y}+\pi$; las imágenes presentan similares características geométricas aunque se aprecia que con el incremento del número de franjas el borde oscuro en la fase envuelta tiende a ser menos anguloso. En la Figura 6 el límite superior de frecuencia de las franjas a detectar, esta limitado por la discretización del CMOS o el tamaño del pixel que debe discernir entre una franja y otra. 

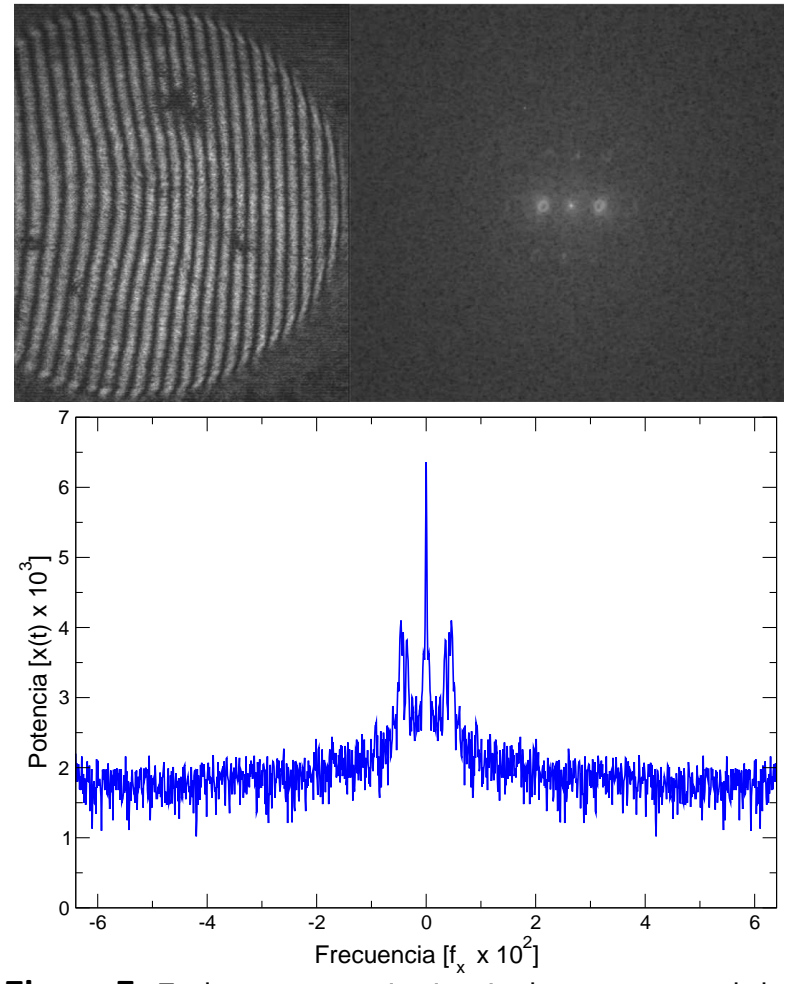

Figura 7: En la parte superior izquierda se muestra el dominio espacial del interferograma del espejo cóncavo M2 y a la derecha su transformada de Fourier bidimensional; en la parte inferior se muestra un perfil central de la transformada de Fourier.

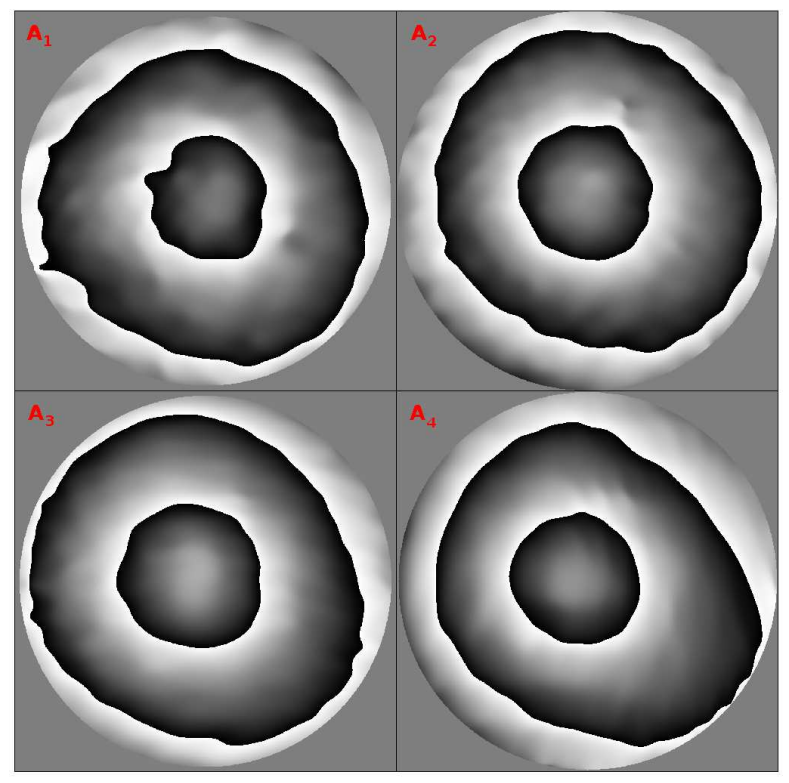

Figura 8: Fase envuelta de los interferogramas con 6, 13, 29 y 39 franjas.
Para obtener la fase desenvuelta se han desarrollado algoritmos [6] que corrigen los cambios bruscos de fase en los píxeles vecinos debido a que se puede estar en presencia de singularidades. La importancia en la construcción del algoritmo es reducir en un mínimo los errores durante el desenvolvimiento y no perder información de la muestra.

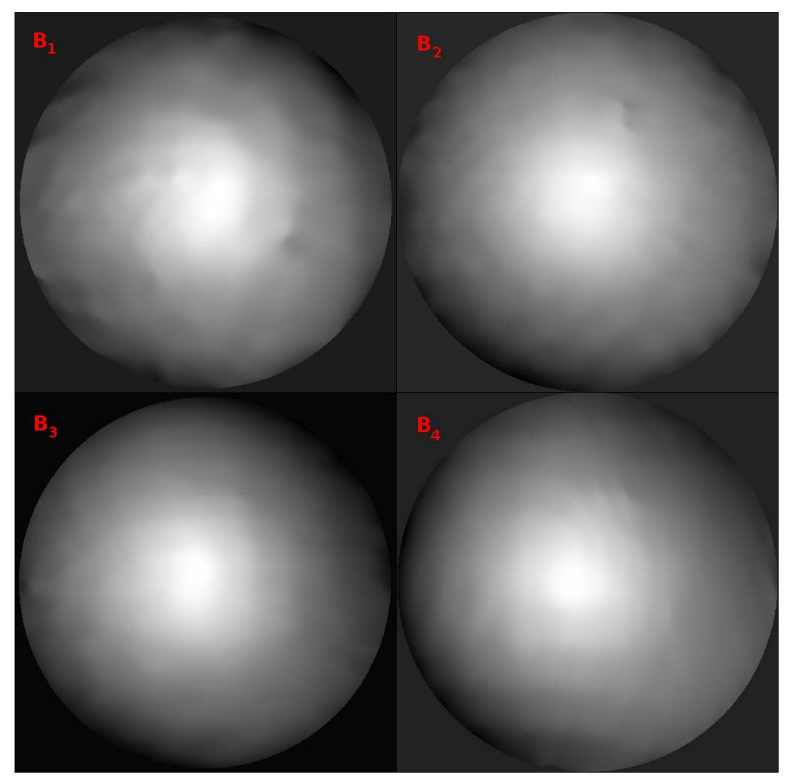

Figura 9: Desenvolviento las fases para 6 (B1), 13 (B2), 29 (B3) y 39 (B4) franjas de interferencias.

En la Figura 9 se muestra la fase desenvuelta obtenidos a partir de las imagenes mostradas en la Figura 8 a las que se aplicaron el método Itoh segun el criterio de las Ec.(12) y Ec.(13). $B_{1}, B_{2}, B_{3}$ y $B_{4}$ muestran los valores de fase continua para los interferogramas de 6 , 13, 29 y 39 franjas; en todas las imágenes se muestra una zona brillante en la parte central lo que nos lleva a inferir una elevación en esa sección, sin importar la cantidad de franjas el resultado es muy similar.

El algoritmo para desenvolver la fase, primero desenvuelve secuencialmente las filas de la imagen envuelta produciendo una intermedia parcialmente desenvuelta; luego esta última fue nuevamente desenvuelta a lo largo de las columnas, obteniendo la imagen de fase continua que se muestran en la Figura 9. El error $(h)$ en la forma superficial del espejo cóncavo se obtiene aplicando la Ec.(14). y la diferencia de pico-valle de los cuatro interferogramas experimentales se encuentran entre $1.11 \lambda$ y $1.25 \lambda$, Figura 10 . 

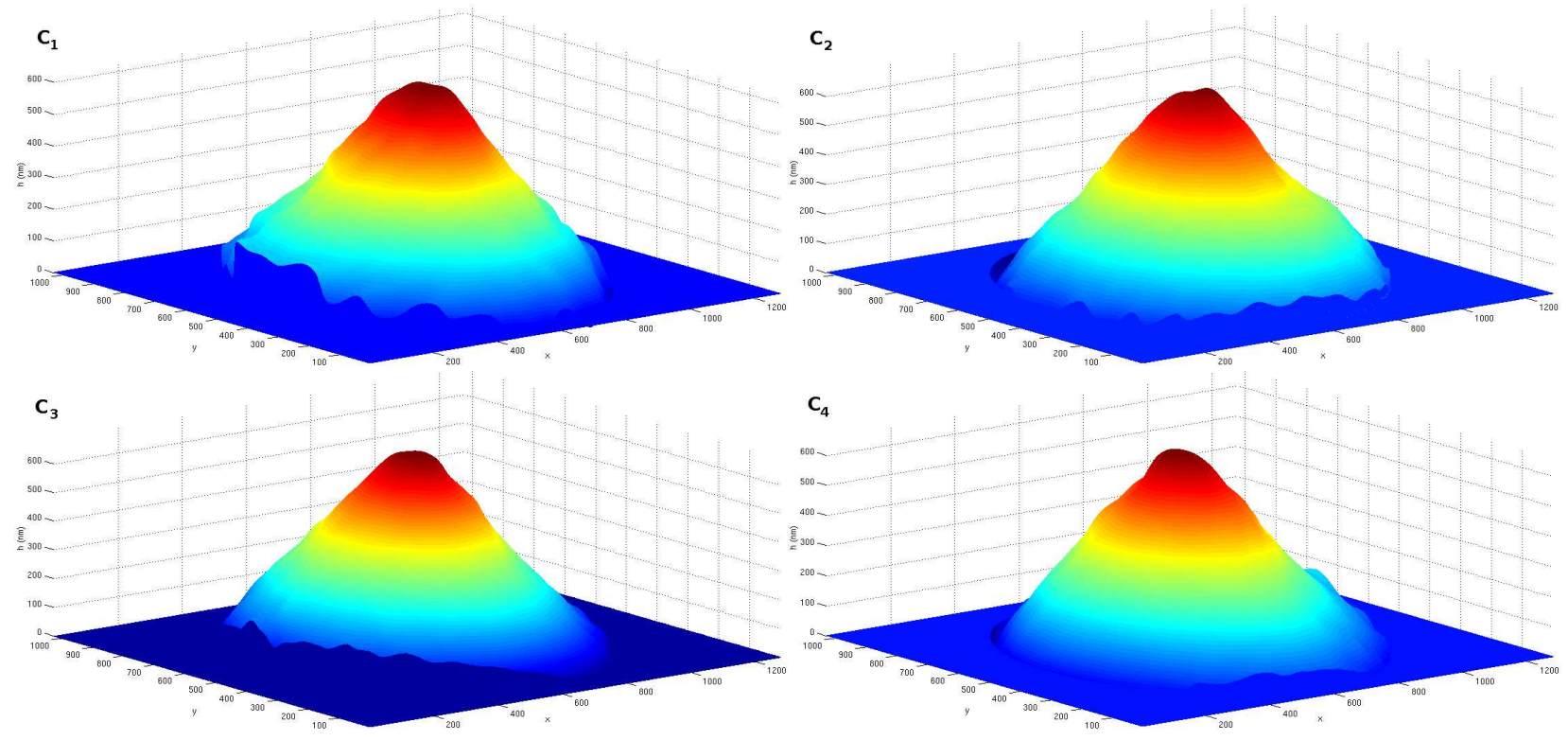

Figura 10: Gráficos 3D de la elevación de la superficie del espejo cóncavo M2 calculadas de los interferogramas con 6, 13 (parte superior), 29 y 39 franjas (parte inferior), con una diferencia de pico-valle que abarca de $1.11 \lambda$ a $1.25 \lambda$.

\section{Conclusiones}

La extracción de la fase consiste básicamente en analizar una imagen de franjas con el uso de la transformada de Fourier filtrándola del ruido y de la frecuencia de la portadora, el resultado es la fase envuelta en el intervalo $-\pi,+\pi$. Para conseguir la fase desenvuelta fue necesario el desarrollo de un algoritmo basado en el método de Itoh.

Se obtuvieron resultados experimentales de la calidad de la superficie cóncava de un espejo por la técnica de interferometría aplicando la transformada de Fourier. Fueron analizados cuatro interferogramas de diferentes números de franjas; la superficie en todos los casos muestra una elevación o protuberancia en la zona central cuyo valor oscila entre $1.11 \lambda$ y $1.25 \lambda$. Este método es aplicable a otros tipo de elementos ópticos como lentes y prismas ya que solo se requiere de un laser, lentes, diafragmas, iris y una cámara de adquisición de imagenes para que la automatización del análisis de la superficie se pueda realizar a tiempo real.

El método de Itoh fue suficiente para desenvolver la fase. Pero, en algunos casos, este proceso puede ser una tarea muy difícil de lograr ya que un error en el procesamiento de un píxel de una imagen, indefinición o discontinuidad de la fase no conocida afecta los siguientes píxeles haciendo que la información de la fase desenvuelta de la imagen sea inutilizable.

\section{Agradecimientos}

Nuestro agradecimiento al personal del Laboratorio de Óptica y Taller de Óptica de la Pontificia Universidad Católica, representados por el profesor Guillermo Baldwin quien nos permitió el acceso a equipos, materiales y muestras que hicieron posible esta investigación.

\section{Referencias}

[1] Daniel Malacara, Alejandro Cornejo, y M. V. R. K. Murty; Bibliography of Various Optical Testing Methods; Appl. Opt. 14, 1065 (1975).

[2] J. Caulfield y William Friday; Bibliography on optical testing; Appl. Opt. 20, 1497 (1981).

[3] Mitsuo Takeda, Hideki Ina y Seiji Kobayashi; Fourier-transform method of fringe-pattern analysis for computer-based topography and interferometry; J. Opt. Soc. Am. 72, 156 (1982).

[4] A. Martínez, J. A. Rayas, J. M. Flores, R. Rodríguez-Vera y D. Donato Aguayo; Técnicas óp- 
ticas para el contorneo de superficies tridimensionales; Rev. Mex. Fís. 51, 431 (2005).

[5] http://www.thorlabs.com/thorProduct.cfm? partNumber=DCC1545M

[6] Dennis C. Ghligia y Mark D. Pritt, Two dimensional phase unwrapping, pp. 311, John Wiley \& sons, New York (1998).
[7] Kazuyoshi Itoh, Analysis of the phase unwrapping algorithm, Appl. Opt. 21, 2470 (1982).

[8] I. Juvelis, S. Vallmitjana, A. Carnicer y J. Campos; The role of amplitude and phase of the Fourier transform in the digital image processing; Am. J. Phys. 59, 744 (1991).

[9] Daniel Malacara, Optical shop testing, pp. 502, John Wiley \& Sons, New York (1992). 